\title{
Glypican-3 expression in malignant small round cell tumors
}

\author{
YUICHI SHIBUI $^{1 *}$, KINA MIYOSHI $^{2 *}$, KENICHI KOHASHI $^{1}$, YOSHIAKI KINOSHITA ${ }^{2}$, \\ MASAAKI KUDA $^{2}$, HIDETAKA YAMAMOTO ${ }^{1}$, TOMOAKI TAGUCHI ${ }^{2}$ and YOSHINAO ODA ${ }^{1}$ \\ Departments of ${ }^{1}$ Anatomic Pathology and ${ }^{2}$ Pediatric Surgery, Graduate School of Medical Sciences, \\ Kyushu University, Fukuoka 812-8582, Japan
}

Received August 7, 2018; Accepted December 12, 2018

DOI: $10.3892 / 01.2019 .9976$

\begin{abstract}
Malignant small round cell tumors usually progress rapidly and show resistance to chemotherapy, and it is often difficult to make a definitive diagnosis based on their histological morphology. Glypican-3 (GPC3) is a highly tumor-specific antigen, and the overexpression of GPC3 was reported in many pediatric and adult malignancies. In the present study, we investigated the GPC 3 expression in pediatric malignant small round cell tumors to assess its role in the differential diagnosis of the tumors. Immunohistochemistry was performed to assess the expression of GPC 3 in samples from 84 rhabdomyosarcomas (RMSs; 44 alveolar and 40 embryonal RMSs), 62 Ewing sarcomas (EWSs), 35 neuroblastomas (NBs) and two desmoplastic small round cell tumors (DSRCTs). We performed a reverse transcription-quantitative polymerase chain reaction for GPC3 to determine the GPC3 mRNA expression in samples from 66 frozen tumors (23 RMSs, 28 EWSs and $15 \mathrm{NBs}$ ). The serum expression levels of GPC3 were analyzed in pre-operative blood samples from two RMS and eight NB patients. In total, 25\% (21/84) of the RMSs and 3\% (1/35) of the NBs exhibited a focal expression of GPC3, whereas, the other specimens showed no GPC3 expression. The GPC 3 mRNA expression level of the RMSs with positive GPC3 expression $(n=6)$ was significantly higher compared with the RMSs without such expression $(n=17)$. A total of two cases of NB showed high serum levels of GPC3, but neither tumor showed immunoreactivity for GPC3. The immunohistochemical overexpression of GPC3
\end{abstract}

Correspondence to: Professor Yoshinao Oda, Department of Anatomic Pathology, Graduate School of Medical Sciences, Kyushu University, 3-1-1 Maidashi, Fukuoka 812-8582, Japan

E-mail: oda@surgpath.med.kyushu-u.ac.jp

*Contributed equally

Key words: glypican-3, small round cell tumors, rhabdomyosarcoma, Ewing sarcoma, desmoplastic small round cell tumor, oncofetal protein may be a candidate ancillary parameter in the differential diagnosis of RMS from EWS and DSRCT.

\section{Introduction}

Glypican-3 (GPC3) is a cell-surface heparin sulfate proteoglycan that binds to the cell surface membrane through a glycosyl-phosphatidylinositol anchor (1). It expresses predominantly during development, tissue- and stage-specifically in the fetal liver, kidney, vertebrae, and genital system, but not in most of the mature normal organs (2). As GPC3 is located in chromosome Xq26, its loss-of-function mutation in the human GPC3 gene results in Simpson-Golabi-Behmel syndrome, which is an X-linked disorder characterized by pre- and postnatal overgrowth that is accompanied by a high risk of the development of embryonal tumors such as hepatoblastoma, neuroblastoma (NB), gonadoblastoma, Wilms tumor, and hepatocellular carcinoma $(3,4)$.

Malignant small round cell tumors are histologically characterized by a proliferation of small round tumor cells with scant cytoplasm, and they are often difficult to distinguish by standard histology or additional immunohistochemistry. However, the quick initiation of treatment is needed for individuals with such tumors.

GPC3 expression was recently reported in several pediatric malignancies as well as in adult malignancies, and thus its potential usefulness as a novel diagnostic marker is being recognized. It is commonly known that GPC3 is expressed in hepatocellular carcinoma, and the expression of GPC 3 in many primary liver tumors has been investigated (5-8). Kinoshita et al examined the GPC3 expression in pediatric malignant solid tumors, and they demonstrated that yolk sac tumor and hepatoblastoma showed a high frequency of GPC 3 expression (9).

In the present study, we immunohistochemically analyzed the GPC3 protein expression in several types of malignant small round cell tumors: Rhabdomyosarcoma (RMS), NB, Ewing sarcoma (EWS), and desmoplastic small round cell tumors (DSRCT). We determined the GPC3 protein expression in order to assess the utility of GPC 3 as a diagnostic marker in these tumors. We also examined the mRNA expression of GPC3 in frozen samples by performing a reverse transcription-quantitative polymerase chain reaction (RT-qPCR) and by determining the serum-soluble GPC3 level in preoperative blood samples with an enzyme-linked immunosorbent assay (ELISA). 


\section{Patients and methods}

Patients and tissue specimens. We collected 183 formalin-fixed paraffin-embedded specimens from the soft-tissue tumor files registered between 1976 and 2007 at the Department of Anatomic Pathology, Graduate School of Medical Sciences, Kyushu University, Fukuoka, Japan (Table I). These samples included 84 RMSs (44 alveolar RMSs and 40 embryonal RMSs), 62 EWSs, 35 NBs, and two DSRCTs. In addition, 86 frozen samples (66 tumor frozen samples and 20 samples of surrounding non-tumorous skeletal muscle from patients who had undergone surgery for various types of sarcoma) were snap-frozen in liquid nitrogen at the time of the surgical procedure and stored at $-80^{\circ} \mathrm{C}$ until use. These 66 frozen tumor samples included 23 RMSs, 28 EWSs and 15 NBs.

We also analyzed the serum levels of GPC3 in preoperative blood samples from 10 patients (eight with NBs and two with RMSs). Our study included the RMS and NB cases from a previous study of GPC 3 expression in pediatric malignant solid tumors by Kinoshita et al (9). The institutional review board at Kyushu University approved this study (approval no. 25-143).

Immunohistochemistry for GPC3. Immunohistochemistry was performed by the streptavidin-biotin-peroxidase method (Histofine; Nichirei, Tokyo) using a mouse monoclonal antibody against GPC3 (clone 1G12, 1:200; BioMosaics, Burlington, VT, USA). We considered the cytoplasmic or membrane staining pattern positive. The immunoreactivity for GPC3 was assessed independently by three pathologists (K.M., K.K., Y.Y.) in a blinded fashion with the use of the labeling index (LI). The GPC3-LI was determined using the number of positively stained tumor cells among $\geq 500$ tumor cells. We classified the protein expression in the tumor tissues into three categories according to the LI percentage: negative, $<1 \%$; focally positive, $1-10 \%$; and positive, $>10 \%$ as described (10).

We categorized the RMS samples into a GPC3-positive and focally positive expression group and a negative-expression group. A log-rank based survival analysis was conducted for the comparison of the overall survival of the patients in the two groups.

TaqMan PCR to detect GPC3 mRNA expression. We performed RT-qPCR for $G P C 3$ and analyzed the results using TaqMan assay reagents (GPC3 Hs00170471_m1; GAPDH Hs99999905_m1) and an ABI Prism 7700 Sequence Detection system (all Applied Biosystems, Foster City, CA, USA). The $\mathrm{PCR}$ reaction was carried out according to the manufacturer's protocol. The obtained data were standardized using data of the international housekeeping gene, GAPDH. All of the reactions for the standard samples and the patients' samples were performed in triplicate. The data were averaged from the values obtained in each reaction. The final numerical value $(\mathrm{V})$ in each sample was calculated as follows: $\mathrm{V}=G P C 3$ mRNA value $\times 10^{6} / G A P D H$ mRNA value.

Detection of the serum-soluble GPC3 by ELISA. Preoperative blood serum samples were available in 10 patients (the eight NB and two RMS patients). The GPC3 serum levels were determined by a commercial ELISA kit (BioMosaics) following the manufacturer's protocol. Based on the data of healthy adult samples with the standard deviation, we set the cut-off level for GPC3 at $178 \mathrm{ng} / \mathrm{ml}$, as described (9).

Statistical analyses. JMP ${ }^{\circledR} 13$ (SAS Institute, Inc., Cary, NC, USA) was used for the statistical analysis. The data regarding the relative expressions of GPC 3 mRNA and protein are presented as the mean \pm standard deviation of at least three independent experiments. Univariate survival analysis was conducted according to the Kaplan-Meier method, and the difference between the survival curves was analyzed with the log-rank test. $\mathrm{P}<0.05$ was considered to indicate a statistically significant difference.

\section{Results}

Immunoreactivity for GPC3. The results of the immunohistochemical analysis are summarized in Table II. In the RMS group, 19.0\% (16/84) showed positive immunoreactivity for GPC3 and 6.0\% (5/84) showed focally positive staining. The embryonal RMSs and alveolar RMSs showed approximately the same proportion of GPC3-positive immunoreactivity. In contrast, none of the EWS or DSRCT cases showed GPC 3 expression. Only a single NB showed GPC3 protein expression (Fig. 1).

Survival analysis of RMS. Survival data were available in 70 of the 84 RMSs (36 ARMSs and 34 ERMSs), and the follow-up periods of these cases ranged from 2 to 254 months (average 62 months). We divided the RMS patients into the immunohistochemically GPC3-positive or focally positive and -negative groups. The survival rate of the negative GPC3 expression group was considerably lower than that of the positive/focally positive group, though the difference was not significant ( $\mathrm{P}=0.58$, Fig. 2).

GPC 3 mRNA expression by TaqMan PCR. Fig. 3 provides the boxplots of GPC3 mRNA expression and the median values according to: the two groups of RMSs with immunohistochemically positive GPC3 expression (615.23), the RMSs with negative GPC3 expression (345.18), the EWSs (12.03), the NBs (43.43), and non-tumorous skeletal muscle (7.47). The GPC3 mRNA expression level of the RMSs with positive GPC 3 expression was significantly higher than those of the EWS, NB, and non-tumorous skeletal muscle groups $(\mathrm{P}=0.0003,0.0024$ and 0.0004 , respectively).

Serum-soluble GPC3 protein level. Regarding the malignant small round cell tumors' serum-soluble GPC3 protein levels, our analyses revealed that although neither of the tumors showed immunoreactivity for GPC3, three cases of NBs showed high serum levels of GPC3.

\section{Discussion}

GPC3 is widely known to express in many malignancies such as embryonal tumors (Wilms tumor, hepatoblastoma, and NB) (11), germ cell tumors (yolk sac tumor, immature teratoma, and embryonal carcinoma) (12), carcinomas 
Table I. Clinical characteristics of malignant small round cell tumors.

\begin{tabular}{|c|c|c|c|c|c|}
\hline Characteristics & $\begin{array}{l}\text { Alveolar } \\
\text { rhabdomyosarcoma, } \mathrm{n}=44\end{array}$ & $\begin{array}{c}\text { Embryonal } \\
\text { rhabdomyosarcoma, } n=40\end{array}$ & $\begin{array}{c}\text { Ewing } \\
\text { sarcoma, } n=62\end{array}$ & Neuroblastoma, $\mathrm{n}=35$ & $\begin{array}{l}\text { Desmoplastic } \\
\text { round cell } \\
\text { tumors, } n=2\end{array}$ \\
\hline \multicolumn{6}{|l|}{ Sex } \\
\hline Male & 23 & 19 & 27 & 20 & 2 \\
\hline Female & 21 & 21 & 35 & 15 & 0 \\
\hline \multicolumn{6}{|l|}{ Age, years } \\
\hline$<15$ & 22 & 32 & 26 & 35 & 1 \\
\hline$\geq 15$ & 22 & 8 & 36 & 0 & 1 \\
\hline
\end{tabular}

Table II. Glypican 3 expression in small round cell tumors.

\begin{tabular}{lccc}
\hline $\begin{array}{l}\text { Small round cell } \\
\text { tumor subtype }\end{array}$ & $\begin{array}{c}\text { GPC3 } \\
(+)(\%)\end{array}$ & $\begin{array}{c}\text { GPC3 } \\
( \pm)(\%)\end{array}$ & $\begin{array}{c}\text { GPC3 } \\
(-)(\%)\end{array}$ \\
\hline RMS, n=84 & $16(19.0)$ & $5(6.0)$ & $63(75.0)$ \\
Alveolar RMS, $\mathrm{n}=44$ & $8(18.2)$ & $1(2.3)$ & $35(79.5)$ \\
Embryonal RMS, $\mathrm{n}=40$ & $8(20.0)$ & $4(10.0)$ & $28(70.0)$ \\
EWS, n=62 & 0 & 0 & $62(100)$ \\
NB, $\mathrm{n}=35$ & $1(2.9)$ & 0 & $34(97.1)$ \\
DSRCT & 0 & 0 & $2(100)$ \\
\end{tabular}

+ , positive; \pm , focally positive; -, negative; RMS, rhabdomyosarcoma; EWS, Ewing sarcoma; NB, neuroblastoma; DSRCT, desmoplastic small round cell tumor; GPC3, glypican-3.

(hepatocellular carcinoma and pulmonary squamous cell carcinoma) $(6,13)$, sarcomas (malignant rhabdoid tumor and RMS) $(10,14)$ and malignant melanoma (15). In the present study, we observed the overexpression of GPC3 in $25 \%$ (21/84) of the RMSs but no expression in the EWSs, which was similar to the results reported by Thway, who described the frequency of GPC3 immunoreactivity among 213 RMSs as $34.7 \%(n=74)(14)$.

Our observations of a high expression of GPC3 mRNA in RMSs and a low expression in EWSs and DSRCTs are similar to the results reported by Baer et al (16). Therefore, the evaluation of GPC3 expression may have the potential to be an ancillary parameter in the differential diagnosis of RMS from EWS and DSRCT.

Some of the GPC3-negative RMSs in the present analysis showed a high expression of GPC3 mRNA. We speculate that GPC3 protein expression may be regulated by other post-transcriptional regulatory mechanisms such as microRNA.

Studies by Nakatsura et al revealed the high expression of GPC3 mRNA and protein in hepatocellular carcinoma and melanoma $(6,15)$. They also noted that GPC3 peptides may be suitable for cancer immunotherapy for GPC3-expressing tumors, because of its low expression in most other normal tissues in adults. Nakatsura's group reported the results of a phase I clinical trial regarding the immune and clinical responses of advanced hepatocellular carcinoma (HCC) patients treated with a GPC3-derived peptide vaccine (17).
In our present series, approx. $20 \%$ of the 29 cases of ARMS, known as a poor-prognosis malignancy, showed positivity for GPC3 and a high GPC3 mRNA level in tumor tissue. Thus, GPC3 peptide immunotherapy may have a beneficial role for ARMS patients.

Several studies revealed the poorer prognosis of HCC patients with GPC3 overexpression compared to those with no or low GPC3 expression $(18,19)$. GPC3 is necessary for HCC cell growth, adhesion, and migration by upregulating canonical Wnt signaling and insulin-like growth factor-2 in vitro (20). In the present study, we assessed the prognosis of RMS with the positive vs. negative status of GPC3 expression in tumor tissue, and the results of our analyses showed a tendency for a poor prognosis in the negative status, but our findings did not reveal a significant prognostic difference between the two groups. The cause of the difference between the prognosis of HCC and that of RMS is unclear. GPC3 expression may thus have limited use as a prognostic indicator in RMS.

It was a predictable result that most of our NB cases $(n=35)$ showed no GPC3 immunoreactivity, and also showed low GPC3 mRNA expression levels. Chan et al reported that the immunohistochemical GPC3 expression was entirely negative in clinical samples of NB (21), whereas Saikali and Sinnett reported increased GPC3 mRNA expression levels in most of the 10 NB cell lines they examined (11). Further studies using a larger number of $\mathrm{NB}$ cases are required to confirm the correlation between GPC3 immunoreactivity and the mRNA expression level.

The serum level of GPC3 was proposed as a novel tumor marker for HCC, along with its significant correlations with the protein and mRNA expression levels (6). We observed high GPC3 serum levels in three present NB patients, although these patients had immunohistochemically GPC3-negative tumors. It is unclear what caused this contradiction. The usefulness of the serum level of GPC3 as a novel marker was not clarified in the present study. Investigations of larger numbers of cases are necessary to test its applicability.

In summary, our findings demonstrated GPC3 overexpression in RMS, whereas other malignant small round cell tumors (i.e., EWS, DSRCT, and most of the NB cases) showed no GPC3 expression. This difference in expression status could be useful in the differential diagnosis of small round cell tumors. GPC3 immunotherapy could be a beneficial approach for RMSs with GPC3 overexpression. 
A

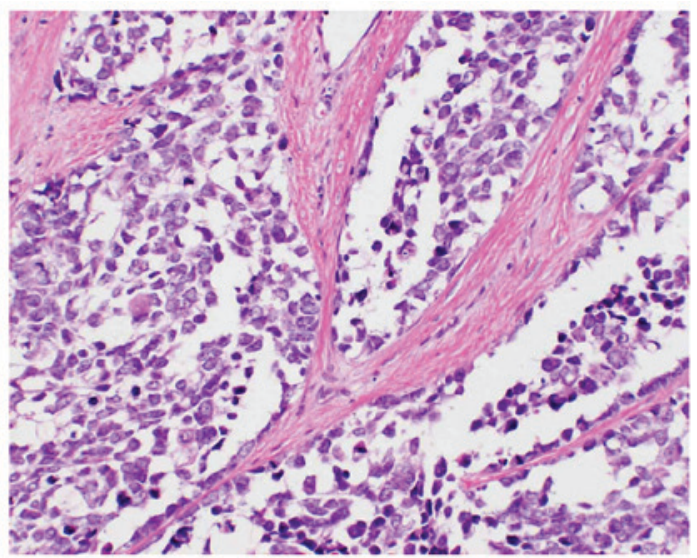

C

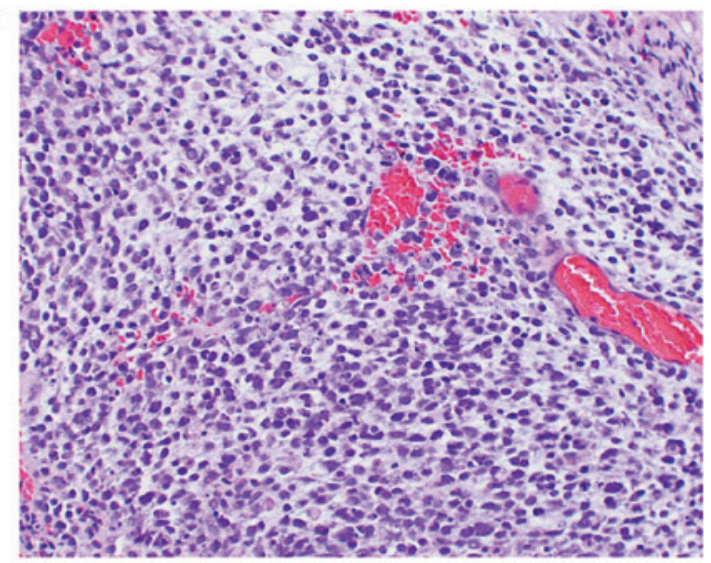

E

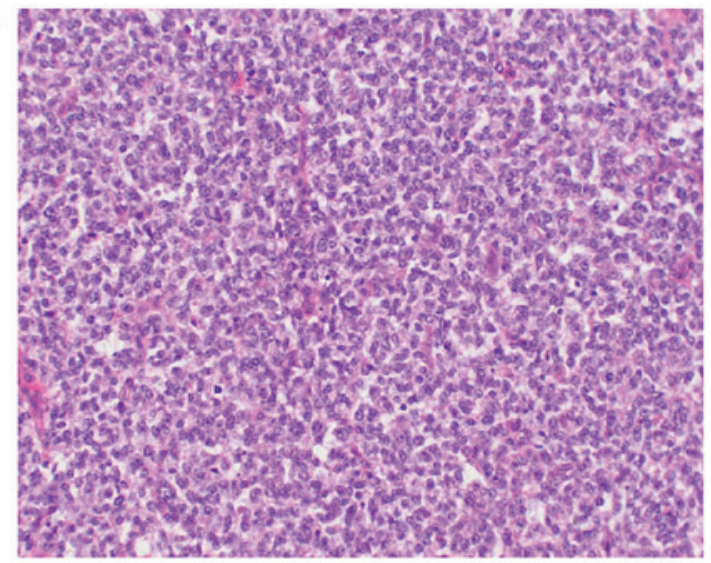

G

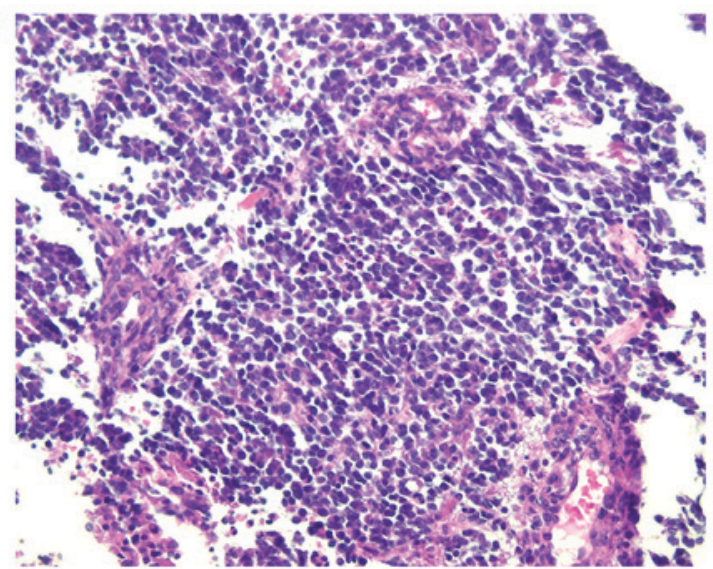

B

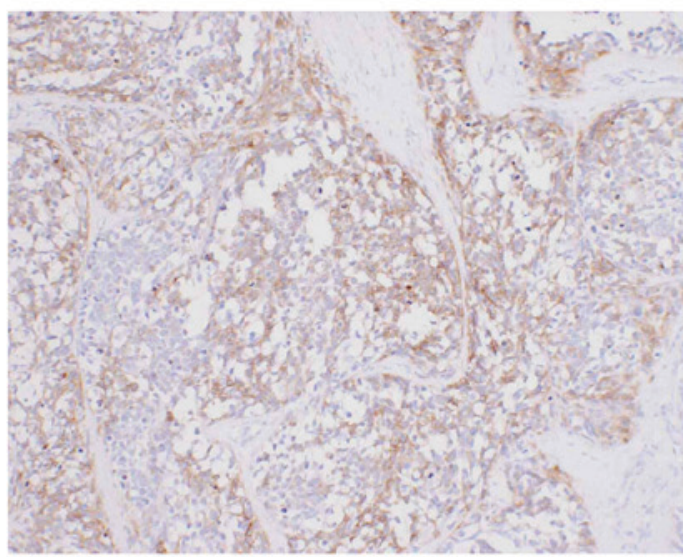

D

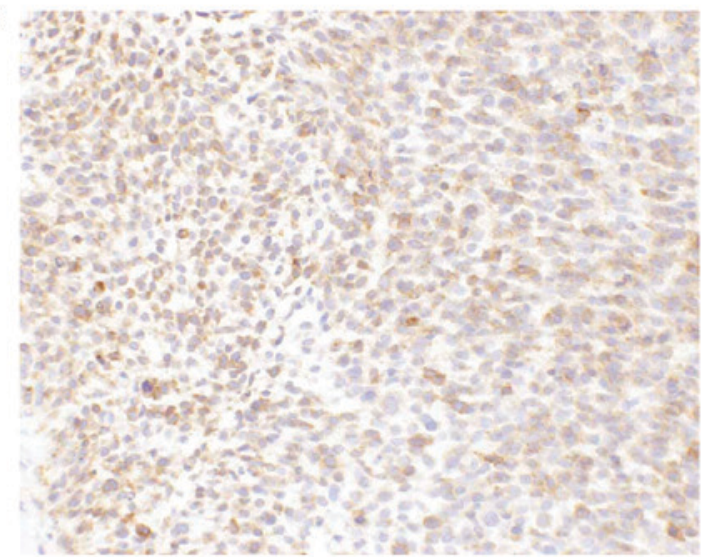

F

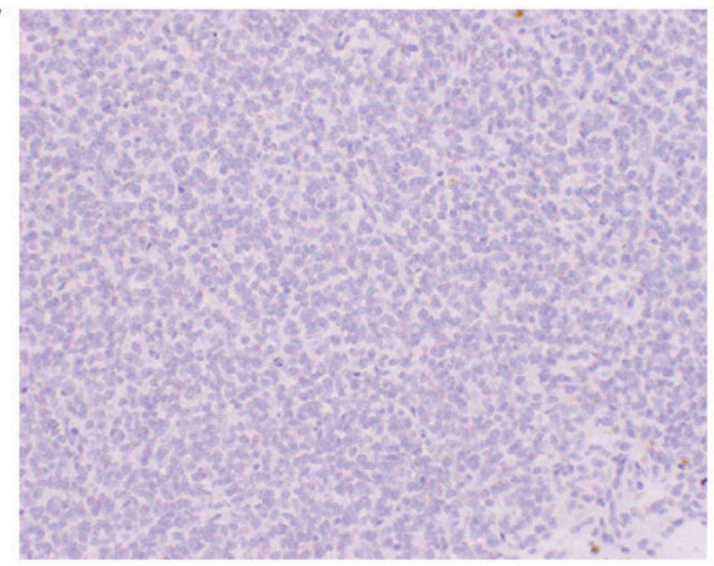

H

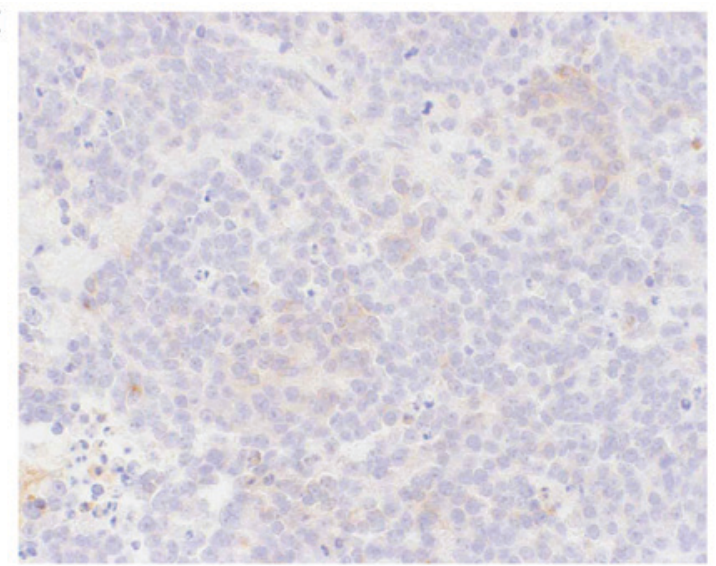

Figure 1. Hematoxylin and eosin histologic and GPC3 immunohistochemical findings. (A and B) Alveolar rhabdomyosarcoma (6-year-old male). (C and D) Embryonal rhabdomyosarcoma (6-year-old male); tumor cells were positive for GPC3. (E and F) Ewing sarcoma (5-year-old male); tumor cells showed no GPC3 expression. (G and H) Neuroblastoma (1-year-old male); only this NB case showed focal GPC3 expression (original magnification, x200). GPC3, glypican-3; NB, neuroblastoma. 


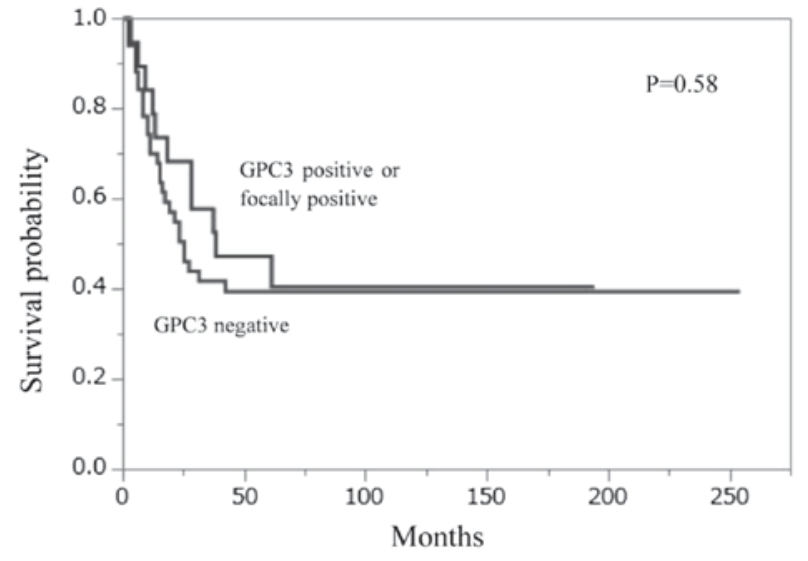

Figure 2. Overall survival analysis of the RMS patients. There was no significant difference between the survival rates of the GPC3 expression-negative and -positive groups $(\mathrm{P}=0.58)$. GPC3, glypican-3; RMS, rhabdomyosarcoma.

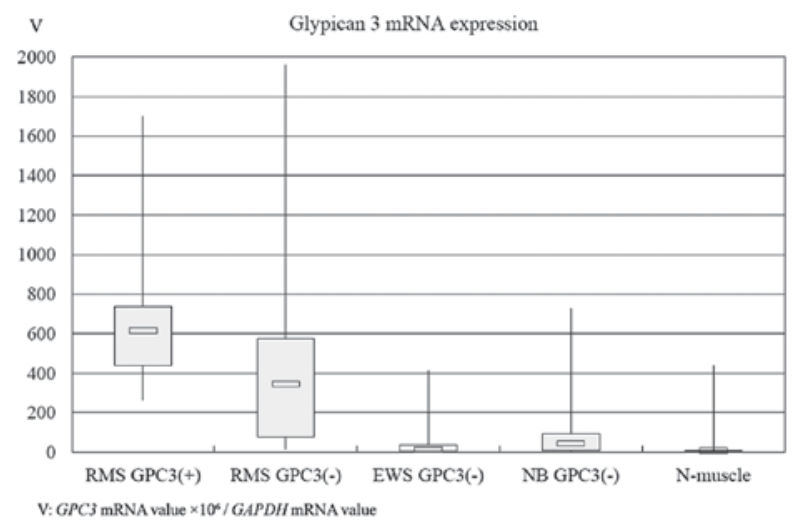

Figure 3. Boxplot of GPC3 mRNA expression. Values listed beside each boxplot are the median values of the GPC3 mRNA expression levels. The median value of the RMS with GPC 3 overexpression group was significantly higher than those of the EWS, NB and non-tumorous skeletal muscle groups by Mann-Whitney U-test $(\mathrm{P}=0.0003,0.0024$ and 0.0004 , respectively). GPC3, glypican-3; RMS, rhabdomyosarcoma; EWS, Ewing sarcoma; NB, neuroblastoma.

\section{Acknowledgements}

Not applicable.

\section{Funding}

The present study was supported by the Japan Society for the Promotion of Science KAKENHI (grants no. 25293088 and 26460435).

\section{Availability of data and materials}

All data generated or analyzed during this study are included in this published article.

\section{Authors' contributions}

YS, KM, KK, HY and YO designed the study. YS, KM and MK performed the experiments. YK and TT collected the clinical information. YS and KM wrote the manuscript. KK,
HY and YO reviewed and checked the manuscript. All authors read and approved the final manuscript.

\section{Ethics approval and consent to participate}

The present study was approved by the Kyushu University Committee of Bioethics (approval no. 29-429; 2017).

\section{Patient consent for publication}

Not applicable.

\section{Competing interests}

The authors declare that there are no competing interests.

\section{References}

1. Filmus J: Glypicans in growth control and cancer. Glycobiology 11: 19R-23R, 2001.

2. Iglesias BV, Centeno G, Pascuccelli H, Ward F, Peters MG, Filmus J, Puricelli L and de Kier Joffé EB: Expression pattern of glypican-3 (GPC3) during human embryonic and fetal development. Histol Histopathol 23: 1333-1340, 2008.

3. DeBaun MR, Ess J and Saunders S: Simpson Golabi Behmel syndrome: Progress toward understanding the molecular basis for overgrowth, malformation, and cancer predisposition. Mol Genet Metab 72: 279-286, 2001.

4. Lapunzina P: Risk of tumorigenesis in overgrowth syndromes: A comprehensive review. Am J Med Genet C Semin Med Genet 137C: 53-71, 2005.

5. Schirwani S, Novelli A, Digilio MC, Bourn D, Wilson V, Roberts C, Dallapiccola B and Hobson E: Duplications of GPC3 and GPC4 genes in symptomatic female carriers of simpson-golabi-behmel syndrome type 1 . Eur J Med Genet pii: S1769-7212: 30203-30209, 2018.

6. Nakatsura T, Yoshitake Y, Senju S, Monji M, Komori H, Motomura Y, Hosaka S, Beppu T, Ishiko T, Kamohara H, et al: Glypican-3, overexpressed specifically in human hepatocellular carcinoma, is a novel tumor marker. Biochem Biophys Res Commun 306: 16-25, 2003.

7. Levy M, Trivedi A, Zhang J, Miles L, Mattis AN, Kim GE, Lassman C, Anders RA, Misdraji J, Yerian LM, et al: Expression of glypican-3 in undifferentiated embryonal sarcoma and mesenchymal hamartoma of the liver. Hum Pathol 43: 695-701, 2012.

8. Xing XL, Qin H, Yan SQ, Zhou LS, Chen P and Zhao D: Expression of glypican-3 is highly associated with pediatric hepatoblastoma: A systemic analysis. Asian Pac J Cancer Prev 16: 1029-1031, 2015.

9. Kinoshita Y, Tanaka S, Souzaki R, Miyoshi K, Kohashi K, Oda Y, Nakatsura $\mathrm{T}$ and Taguchi T: Glypican 3 expression in pediatric malignant solid tumors. Eur J Pediatr Surg 25: 138-144, 2015.

10. Kohashi K, Nakatsura T, Kinoshita Y, Yamamoto H, Yamada Y, Tajiri T, Taguchi T, Iwamoto Y and Oda Y: Glypican 3 expression in tumors with loss of SMARCB1/INI1 protein expression. Hum Pathol 44: 526-533, 2013.

11. Saikali Z and Sinnett D: Expression of glypican 3 (GPC3) in embryonal tumors. Int J Cancer 89: 418-422, 2000.

12. Ota S, Hishinuma M, Yamauchi N, Goto A, Morikawa T, Fujimura T, Kitamura T, Kodama T, Aburatani $\mathrm{H}$ and Fukayama M: Oncofetal protein glypican-3 in testicular germ-cell tumor. Virchows Arch 449: 308-314, 2006.

13. Aviel-Ronen S, Lau SK, Pintilie M, Lau D, Liu N, Tsao MS and Jothy S: Glypican-3 is overexpressed in lung squamous cell carcinoma, but not in adenocarcinoma. Mod Pathol 21: 817-825, 2008.

14. Thway K, Selfe J, Missiaglia E, Fisher C and Shipley J: Glypican-3 is expressed in rhabdomyosarcomas but not adult spindle cell and pleomorphic sarcomas. J Clin Pathol 64: 587-591, 2011.

15. Nakatsura T, Kageshita T, Ito S, Wakamatsu K, Monji M, Ikuta Y, Senju S, Ono T and Nishimura Y: Identification of glypican-3 as a novel tumor marker for melanoma. Clin Cancer Res 10: 6612-6621, 2004. 
16. Baer C, Nees M, Breit S, Wakamatsu K, Monji M, Ikuta Y, Senju S, Ono T and Nishimura Y: Profiling and functional annotation of mRNA gene expression in pediatric rhabdomyosarcoma and Ewing's sarcoma. Int J Cancer 110: 687-694, 2004.

17. Sawada Y, Yoshikawa T, Nobuoka D, Shirakawa H, Kuronuma T, Motomura Y, Mizuno S, Ishii H, Nakachi K, Konishi M, et al: Phase I trial of a glypican-3-derived peptide vaccine for advanced hepatocellular carcinoma: Immunologic evidence and potential for improving overall survival. Clin Cancer Res 18: 3686-3696, 2012.

18. Shirakawa H, Suzuki H, Shimomura M, Kojima M, Gotohda N, Takahashi S, Nakagohri T, Konishi M, Kobayashi N, Kinoshita T and Nakatsura T: Glypican-3 expression is correlated with poor prognosis in hepatocellular carcinoma. Cancer Sci 100: 1403-1407, 2009.
19. Zhang J, Zhang M, Ma H, Song X, He L, Ye X and Li X: Overexpression of glypican-3 is a predictor of poor prognosis in hepatocellular carcinoma: An updated meta-analysis. Medicine (Baltimore) 97: e111130, 2018.

20. Midorikawa $\mathrm{Y}$, Ishikawa $\mathrm{S}$, Iwanari $\mathrm{H}$, Imamura T, Sakamoto $\mathrm{H}$, Miyazono K, Kodama T, Makuuchi M and Aburatani H: Glypican-3, overexpressed in hepatocellular carcinoma, modulates FGF2 and BMP-7 signaling. Int J Cancer 103: 455-465, 2003.

21. Chan ES, Pawel BR, Corao DA, Venneti S, Russo P, Santi M and Sullivan LM: Immunohistochemical expression of glypican-3 in pediatric tumors: An analysis of 414 cases. Pediatr Dev Pathol 16: 272-277, 2013. 